\title{
¿Por qué te peleas? Conflictos motores y emociones negativas en la clase de Educación Física: el caso de los juegos de oposición
}

Why are you fighting? Motor conflicts and negative emotions in the physical education class: The case of opposition games

\author{
Unai Sáez de Ocáriz Granja* \\ usaez@gencat.cat \\ Pere Lavega Burgués** \\ plavega@inefc.es \\ Francisco Lagardera Otero** \\ flagardera@inefc.es \\ Antoni Costes Rodríguez** \\ tcostes@inefc.es \\ Jorge Serna Bardavío*** \\ Jorgesema1979@gmail.com \\ * Universidad de Barcelona, España \\ **Universidad de Lleida, España \\ ***Universidad San Jorge, España
}

\section{Resumen:}

Los conflictos, habituales en las escuelas y motivo de preocupación docente e institucional, son situaciones que emergen cuando el alumnado no es capaz de gestionar las emociones negativas. La Educación Física dispone de diferentes familias de juegos motores, como los juegos de oposición, para educar las relaciones interpersonales de los alumnos. Este artículo presenta dos investigaciones que examinaron los efectos de los juegos de oposición en la aparición de conflictos motores en un grupo de 43 estudiantes de educación infantil y primaria (estudio 1) y en la

\begin{abstract}
:
Conflicts, a common phenomenon in schools and a source of concern for teachers and educational authorities, arise when students are unable to deal with their negative emotions. In the context of physical education there are several families of motor games, such as opposition games, that can be used to teach interpersonal relationships among students. This article describes two studies that examined the impact of opposition games in relation to the emergence of conflicts in a group of 43 primary school students (study 1) and the effect of such games on the ex-
\end{abstract}


¿Por qué te peleas? Conflictos motores y emociones negativas en la clase de Educación Física: el caso de los juegos de oposición

Unai Sáez de Ocáriz Granja, Pere lavega Burgués, Francisco lagardera Otero, Antoni

Costes Rodríguez y Jorge Serna Bardavío

vivencia de emociones negativas en un grupo de 220 estudiantes de ESO y BachiIlerato (estudio 2). Los análisis estadísticos (análisis descriptivo y análisis inferencial por medio del modelo de regresión logística univariable y multivariable en el primer estudio y uso de los árboles de clasificación CHAID en el segundo) identificaron que las variables competición y género deben tenerse en cuenta al introducir este tipo de juegos en el currículum escolar. La presencia de competición acentúa los conflictos y la intensidad de las emociones negativas. Además, los chicos tienden a protagonizar mayor cantidad e intensidad de conflictos y de emociones negativas que las chicas, sobre todo ante juegos de oposición competitivos.

\section{Palabras clave:}

Conflicto motor, emociones negativas, juegos de oposición, competición, género. perience of negative emotions in a group of 220 secondary and baccalaureate students (study 2). The statistical analyses (descriptive analysis and inferential analysis by means of univariate and multivariate logistic regression in study 1 , and the use of CHAID classification trees in study 2) showed that the competitive element and gender are variables that should be taken into account when using these kinds of games as part of the school curriculum. The presence of competition heightened conflicts and increased the intensity of negative emotions. Boys tended to be responsible for generating more, and more intense, conflicts, and they also reported more negative emotions than did girls, especially in the context of competitive opposition games.

\section{Key words:}

Motor conflict, negative emotions, opposition games, competition, gender.

\section{Résumé :}

Les conflits, habituels dans les écoles et motif de préoccupation des enseignants et des institutions, sont des situations qui émergent quand les élèves ne sont pas capables de gérer et de contrôler les émotions négatives. Lıéducation physique a des différentes familles de jeux moteurs, comme les jeux d`opposition, pour éduquer les relations interpersonnelles des étudiants. Cet article présente deux études qui ont examiné les effets des jeux d'opposition dans l'apparition des conflits moteurs dans un groupe de 43 élèves de l'école maternelle et de l'enseignement primaire (étude 1), et sur l'expérience des émotions négatives dans un groupe de 220 étudiants de l'école secondaire (ESO et Bachillerato) (étude 2). Lıanalyse statistique (analyse descriptive et lıanalyse dinférence avec un modèle de régression logistique univariée et multivariée dans la première étude, et Isutilisation des arbres de classification CHAID dans le second) a identifié que les variables de compétition et de genre doivent être considérées pour introduire ce type de jeux dans le curriculum scolaire. D'un côté, la présence de la compétition accentue le conflit et lıintensité des émotions négatives. D'autre côté, les garçons ont la tendance à être les protagonistes dans un plus grand nombre de conflits et d'émotions négatives que les filles, en particulier dans les jeux d`opposition compétitifs.

\section{Mots-clés :}

Conflit moteur, émotions négatives, jeux d’opposition, compétition, genre.

Fecha de recepción: 30-12-2013

Fecha de aceptación: 30-1-2014 


\section{Introducción}

Vivimos en el momento de la civilización humana menos agresivo y violento de toda la historia conocida (Elías, 1987), sin embargo, en el contexto educativo, tanto en el marco familiar como en el escolar, se manifiestan situaciones constantes de conflictos de intereses entre los padres y los educadores respecto de los hijos y alumnos.

La Educación Física (EF), a través de la prácticas motrices, ofrece al alumnado la posibilidad de 'aprender a hacer' en un contexto real lo más próximo posible al que se desarrolla en su vida cotidiana. Teniendo en cuenta esta característica o modo específico de aprender, resulta necesario poner a la emoción en el centro de atención de la EF, teniendo en cuenta los rasgos personales de cada alumno, incluyendo sus tendencias emocionales, al seleccionar los contenidos prácticos a proponer si se pretende abordar con coherencia y pertinencia los conflictos generados en el seno de estas clases.

Es por esto que la presente contribución pretende mostrar la relación existente entre los conflictos surgidos en las clases de EF y la vivencia de emociones negativas, muy especialmente cuando se ponen en práctica juegos de oposición competitivos.

\section{Conflictos y emociones en Educación Física}

En cualquier circunstancia de la vida, las personas experimentan constantemente estados emocionales de mayor o menor intensidad, que pueden ser de carácter positivo o negativo, en función de si aporta o no bienestar subjetivo (Bisquerra, 2000; Lazarus, 2000). Los conflictos están directamente relacionados con las emociones, de modo que aparecen en aquellas situaciones en las que las personas experimentan emociones negativas que no son capaces de gestionar de manera autorregulada (Bodtker y Jameson, 2001).

El conflicto es producto de una incompatibilidad entre dos partes, manifestado en un desacuerdo, y con posibilidad de ser transformado por las personas implicadas (Lederach, 1995). Las personas con pocas habilidades sociales suelen tener dificultades para regular las emociones negativas que resultan de la convivencia con otras personas, de ahí la necesidad de abordar este reto de manera compartida por parte de todos los agentes. 
¿Por qué te peleas? Conflictos motores y emociones negativas en la clase de Educación Física: el caso de los juegos de oposición

Unal Sáez de Ocáriz Granja, Pere Lavega Burgués, Francisco lagardera Otero, Antoni Costes Rodríguez y Jorge Serna Bardavío

Desde un punto de vista pedagógico, los conflictos y las emociones son una oportunidad para educar las relaciones interpersonales y transformar la experiencia de vivir en sociedad en un proceso satisfactorio que aporte bienestar subjetivo (París, 2005).

Para educar desde la transformación social y emocional del conflicto (Sáez de Ocáriz, 2011) es preciso conseguir que las tareas motrices y juegos motores propuestos sean significativas para el alumnado; es decir, que se sientan atraídos por el desafío que les plantea este tipo de experiencias motrices. Cuando el alumno consigue fluir al resolver un problema motor, se puede afirmar que éste tiene una intervención orientada a la tarea (Cervelló, Moreno, Alonso e Iglesias, 2006; Digelidis, Papaioannou, Laparidis, y Christodoulidis, 2003; Jiménez, Cervelló, Santos-Rosa y Del Villar, 2006; Newton, Duda y Yin, 2000).

La aparición de conductas conflictivas suele estar asociada a la impulsividad, la ira, la intolerancia, a la frustración y a la falta de empatía (Ramírez-Fernández, 2006). Así mismo, la falta de competencias sociales puede llevar a descifrar incorrectamente las señales de los demás (Carney y Merrel, 2001) originando una lectura emocional equivocada. Esto repercute negativamente en la vida académica del alumnado y también puede ser motivo del desarrollo de comportamientos antisociales (Mestre, Guill, Lopes, Salovey y Olarte, 2006; Petrides, Frederickson y Furnham, 2004). En cambio, los alumnos que son capaces de cooperar y disfrutar en las relaciones interpersonales, experimentan emociones positivas que favorecen la convivencia y la vida académica en la escuela (Baron, 1990; Desivilya y Yagil,2005).

En la EF el juego motor es un contenido especialmente indicado para favorecer la transformación social y emocional del conflicto ya que actúa como laboratorio de relaciones interpersonales de modo que de acuerdo a las reglas o condiciones de la acción motriz, orienta a los alumnos a establecer diferentes tipos de interacciones sociales (Parlebas, 2001).

Cada juego dispone de una lógica interna o patrón de organización interno que establece los modos de relacionarse con el espacio, con el material, con el tiempo y con los demás (Lagardera y Lavega, 2005). Cualquier alumno que participa de un juego interpreta su lógica interna y protagoniza conductas motrices para intentar alcanzar el objetivo propuesto. El concepto de conducta motriz implica considerar el comportamiento significante de la persona, es decir, que saltar, chutar una 
pelota o capturar a un adversario además de ser una intervención motriz, también es una intervención que activa de manera unitaria las diferentes dimensiones de su personalidad: biológica, emocional, cognitiva y social (Parlebas, 2001).

Ante un mismo juego, cada alumno pone en acción conductas motrices únicas y singulares para adaptarse e interpretar la lógica interna de ese juego. Las conductas motrices pueden ser de tres tipos: ajustadas (cuando la respuesta va en la misma dirección a lo que solicita la lógica interna del juego, por ejemplo, pasar bien el balón a un compañero), desajustada (cuando se desvía de esa lógica interna, por ejemplo, pasar el balón al compañero de modo incorrecto de manera intencionada o no) o perversa (cuando se dan respuestas no permitidas por las reglas del juego, por ejemplo, al pasar el balón con las manos en lugar de con los pies). Además, también se pueden presentar conductas verbales de pacto (ajustadas, desajustadas o perversas) cuando los alumnos intentan ponerse de acuerdo para organizarse o solucionar estratégicamente el problema planteado o surgido en el juego (Lagardera y Lavega, 2005).

\section{Tabla 1. Tipos de conductas motrices y verbales conflictivas en Educación}

Física

\begin{tabular}{ll}
$\begin{array}{l}\text { Tipo de } \\
\text { conductas }\end{array}$ & Ejemplos correspondientes al juego del pañuelo \\
\hline $\begin{array}{l}\text { Conductas } \\
\text { Motrices } \\
\text { Ajustadas }\end{array}$ & $\begin{array}{l}\text { Salir al escuchar nuestro número. } \\
\text { Coger el pañuelo y regresar a casa sin ser capturado. } \\
\text { Capturar al adversario cuando ha cogido el pañuelo. }\end{array}$ \\
\hline $\begin{array}{l}\text { Conductas } \\
\text { Motrices } \\
\text { Desajustadas }\end{array}$ & $\begin{array}{l}\text { No salir a tiempo (podría originar gritos y reacciones emocio- } \\
\text { nales inadecuadas sobre el contrario u otros participantes). } \\
\text { Se insulto al no saber regular la rabia o ira de ser eliminado). }\end{array}$ \\
\hline $\begin{array}{l}\text { Conductas } \\
\text { Motrices }\end{array}$ & $\begin{array}{l}\text { Salir cuando se ha mencionado el número de otro compañe- } \\
\text { ro (ante esta trampa se podría originar algún forcejeo entre } \\
\text { los participantes y reacciones de mociones negativas). }\end{array}$ \\
Perversas & $\begin{array}{l}\text { Tras ser capturado pegar un cachete al adversario. } \\
\text { Tras haber sido eliminado un compañero no querer ponerse } \\
\text { Conductas } \\
\text { Verbales de acuerdo con los compañeros de equipo (conducta des- } \\
\text { ajustada) para repartirse los números que quedan entre los } \\
\text { vacto }\end{array}$ \\
$\begin{array}{l}\text { vivos. Esto podría originar una discusión y por tanto reaccio- } \\
\text { nes emocionales conflictivas. }\end{array}$
\end{tabular}

Nota: Tabla basada en Lagardera y Lavega (2005) y Lavega ( 2004) 
¿Por qué te peleas? Conflictos motores y emociones negativas en la clase de Educación Física: el caso de los juegos de oposición

Unal Sáez de Ocáriz Granja, Pere Lavega Burgués, Francisco lagardera Otero, Antoni Costes Rodríguez y Jorge Serna Bardavío

Los conflictos motores se originan cuando un alumno realiza conductas motrices y/o verbales de carácter desajustado o perverso y que afectan directamente a otro participante. Acto seguido provoca una reacción consistente en una agresión verbal, física o mixta (verbal y física simultánea) que precisa de una intervención del profesorado o de los propios protagonistas del conflicto (Sáez de Ocáriz, 2011).

La EF entendida como pedagogía de conductas motrices, puede educar las relaciones interpersonales y la afectividad asociada desde la propia acción motriz; es decir, puede transformar los conflictos sociales y emocionales de los alumnos a partir del propio juego. He aquí uno de los tesoros de esta disciplina pedagógica, aún sin explotar adecuadamente.

Ante este escenario, resulta imprescindible que el profesor se convierta en un experto observador (Costes, 1997) de los comportamientos motores de sus alumnos, teniendo en cuenta a la vez las emociones que los acompañan y sus rasgos biográficos, para poder interpretar las conductas motrices de manera pertinente y justa (Lagardera y Lavega, 2005, 2011). Su intervención podrá consistir en modificar las condiciones de juego para que las reglas orienten a los alumnos hacia otro tipo de relaciones motrices o interviniendo sobre los propios alumnos en busca de un modelo de "ganar-ganar" en el que todas las partes implicadas pueden salir beneficiadas (Burguet, 2003; Sáez de Ocáriz, 2011).

\section{Los conflictos y emociones en los juegos motores de oposición: la competición y el género como variables predictivas}

El profesor de EF dispone de un gran repertorio de juegos motores. Según el tipo de relación motriz que exige la lógica interna de un juego, se identifican cuatro dominios o clases de juegos (Parlebas, 2001): a) juegos psicomotores, donde no existe interacción entre jugadores; b) juegos cooperativos que exigen la ayuda mutua en las interacciones motrices de los participantes; c) juegos de oposición, en la que dos o más protagonistas rivalizan por el objetivo del juego; d) juegos de cooperaciónoposición, en los que varios jugadores cooperan entre ellos y se oponen a un equipo de adversarios.

Este artículo centra la atención en los juegos de oposición, ya que exigen al alumnado activar un nivel elevado de competencias sociales 
y emocionales para adaptarse eficazmente y aceptar de buen grado las relaciones de rivalidad con los otros participantes. El reto es conseguir que el adversario pase de ser visto como persona hostil, a considerarse un agente que nos pone a prueba, para superarnos al tratar de engañar y no ser engañados; de capturar y no ser capturados; de anticiparse y llegar antes que el contrario. La persona orientada hacia el disfrute del propio juego, ve esa experiencia lúdica bajo el modelo "ganar-ganar", donde todos salen enriquecidos, experimentando vivencias emocionales positivas de bienestar personal y social (Alonso, Etxebeste y Lavega, 2010; Alonso, Lavega, y Reche, 2012; Lavega, Filella, Agulló, Soldevilla y March, 2011; Lavega, March, y Filella, 2013; Lee, Mower, Busso, Lee, y Naranyan, 2011).

Sin embargo, si la persona tiene carencias en la relación interpersonal, cada desafío se puede ver como un obstáculo, un estímulo desencadenante de emociones negativas como la frustración, rabia o ira, ante cualquier éxito del rival. La persona se instala en el modelo "ganar-perder" y no es capaz de obtener bienestar cuando no es el ganador (Sáez de Ocáriz, 2011).

Los juegos de oposición pueden realizarse asociados a un marcador final, donde un jugador gana y otro pierde (juegos con estructura de duelo o de una contra uno). También pueden presentarse con otras estructuras de relación (e.g., uno contra todos, todos contra todos) basadas en intercambios de roles sin que exista un desenlace o marcador final (Lagardera y Lavega, 2005).

Los juegos de oposición con marcador pueden desencadenar valores intensos de emociones positivas, pero también valores elevados en emociones negativas. En cambio, si se emplean juegos de oposición sin un resultado final, las emociones positivas no son tan intensas pero tampoco lo son las negativas (Alonso, Gea y Yuste, 2013; Lavega et al. 2013).

Además de estos criterios internos de los juegos, otro aspecto a tener en cuenta son las características de los alumnos, tales como el género, el nivel académico y el entorno sociocultural del que proceden. En las investigaciones que sirven de base para la confección de este artículo, se ha tenido en cuenta el género como variable independiente asociada a los rasgos personales (Lavega et al. 2013).

Desde hace décadas, se vienen realizando estudios en diferentes países europeos (Cerezo, 2009; Defensor del Pueblo, 2007; Díaz-Aguado, 2005; García-Correa, 2001; Ortega, 2002) para observar y detectar cua- 
¿Por qué te peleas? Conflictos motores y emociones negativas en la clase de Educación Física: el caso de los juegos de oposición

Unal Sáez de Ocáriz Granja, Pere Lavega Burgués, Francisco lagardera Otero, Antoni Costes Rodríguez y Jorge Serna Bardavío

les son los factores que influyen en la situaciones conflictivas que se generan en niños y niñas entre los 6 y los 16 años de edad. En dichos trabajos se constata que existe una diferencia significativa entre la manera de actuar de los chicos y de las chicas ante los conflictos. Los chicos se involucran más en los conflictos que las chicas ya que actúan de una manera más violenta y agresiva. Esta misma tendencia se acentúa si los alumnos proceden de una clase social desfavorecida, al estar más expuestos a las relaciones conflictivas.

En base a lo ya descrito, cabe considerar que conocer las características internas de los juegos, así como los rasgos personales de los alumnos, son condiciones necesarias para que el profesor-observador pueda interpretar las conductas motrices de sus alumnos, si pretende ayudar a transformar de manera eficaz la naturaleza social y emocional de los conflictos motores (Sáez de Ocáriz, 2011).

A partir de este marco teórico de referencia, el presente artículo presenta dos investigaciones con el fin de examinar el efecto de la competición en los juegos de oposición (variable interna asociada al juego) y del género (variables externa asociada a los rasgos del alumno) en la aparición de conflictos motores y en la vivencia de emociones en el ámbito escolar.

\section{Método}

\section{Estudio 1: La emergencia de conflictos motores en los juegos de oposición}

\section{PARTICIPANTES}

Este estudio se realizó con 43 estudiantes de un centro de Educación Infantil y Primaria de Lleida (21 niños y 22 niñas; $M=21.5 ; D T=.50)$, con un rango de edad que osciló entre los 8 y los 11 años $(M=9.35$; $D T=1.07)$. De estos participantes, 23 cursaban ciclo medio (11 chicos y 12 chicas) y los 20 restantes estudiaban ciclo superior (10 chicos y 10 chicas). Durante el estudio se garantizaron los aspectos éticos de las investigaciones en las ciencias sociales (Mesía, 2007). 
Procedimientos E INSTRUMENTOS

Esta experiencia formó parte de un programa de educación física destinado a la reducción de la violencia y el absentismo escolar. Dado el perfil del alumnado, de origen socio-económico y cultural altamente desfavorable, el centro educativo llevo a cabo durante dos cursos académicos un plan de autonomía de acción pedagógica a través de actividades de educación física. Se examinaron los conflictos motores detectados a lo largo de 8 sesiones de una unidad didáctica, de una hora dos veces por semana, orientada a educar relaciones interpersonales mediante 32 juegos de oposición. El profesor de educación física se responsabilizó de diseñar el programa de intervención, seleccionar e impartir los juegos (rol docente), para seguidamente, al concluir la sesión, describir de una manera rigurosa, minuciosa y detallada cada uno de los juegos propuestos y los procesos de conflicto motor detectados -rol investigador- (Costes, 1997).

El procedimiento para observar y describir lo acontecido en las clases de educación física fue la observación participante, sin grabación audiovisual para garantizar el anonimato de los alumnos y el sesgo en la investigación. Se empleo como sistema narrativo las notas de campo a través de hojas de observación (Del Rincón, Arnal, Latorre y Sans, 1995), formadas por descripciones concretas y generales del fenómeno observado que se registraron al acabar cada una de las sesiones con el objetivo de explicar los procesos en desarrollo e identificar el modelo de conductas observadas .

\section{ANÁLISIS DE LOS DATOS}

Se realizó un análisis estadístico a través de un análisis descriptivo y un análisis inferencial por medio del modelo de regresión logística univariable y multivariable de los datos cualitativos obtenidos. Se utilizó el paquete estadístico Statistical Package for the Social Sciencies (SPSS) versión 19.0 para Windows.

\section{Estudio 2: La intensidad de las emociones negativas en los juegos de oposición}

PARTICIPANTES

En este estudio participaron 220 estudiantes de ESO y Bachillerato (rango de edad de 12 a 17 años; Medad = 14,3 años, $D T=1,93$ años); 110 
¿Por qué te peleas? Conflictos motores y emociones negativas en la clase de Educación Física: el caso de los juegos de oposición

Unal Sáez de Ocáriz Granja, Pere Lavega Burgués, Francisco lagardera Otero, Antoni Costes Rodríguez y Jorge Serna Bardavío

chicos $(54,10 \%)$ y 119 chicas $(45,90 \%)$, pertenecientes a cinco centros educativos de las comunidades de Cataluña y Valencia: 78 (35,45 \%) del IES Narcís Monturiol de Girona, 68 (30,90 \%) del IES Victoria Kent de Elche, 34 (15,45 \%) del IES Verdaguer de Sant Sadurní de Anoia, Barcelona, $22(10,00 \%)$, del IES Flix de Tarragona y 18 (8,18\%), del IES Betula Alba de Elche. Todos los participantes dieron su consentimiento para participar en este estudio que también fue aprobado por el comité de Ética de la Universidad de Lleida.

\section{Procedimientos E instrumentos}

Selección y aplicación de tareas motrices

Se realizó una sesión de 50 minutos basadas en juegos de oposición. El docente se limitó a plantear los juegos de oposición y seguidamente los participantes procedían a su práctica. Solo se intervino cuando hubo alguna duda sobre la tarea a realizar, así como para gestionar el tiempo de la actividad y organizar el material necesario.

Identificación de la intensidad de las emociones vividas

La intensidad de las emociones experimentadas en cada juego fueron valoradas a través de la escala GES (Games and Emotion Scale), validada por Lavega et al. (2013). En términos de fiabilidad, el valor de alpha de Cronbach's indicó que la consistencia interna fue óptima para toda la escala ( $n=851 ; \alpha=.92$ ). Al finalizar cada tarea, los participantes anotaron en su cuestionario personal del GES el nivel de intensidad (de 0 a 10) que habían experimentado en las trece emociones básicas (Bisquerra, 2000): a) emociones positivas: alegría, humor, amor y felicidad; b) emociones negativas: ira, miedo, ansiedad, tristeza, vergüenza, rechazo; y c) emociones ambiguas: esperanza, sorpresa y compasión. Una puntuación de cero significaba que no había sentido esa emoción, mientras que una puntuación de 10 indicaba que se había vivido con la máxima intensidad.

Análisis de los datos

Se valoró el ajuste a la normalidad de los datos (intensidad de las emociones vividas) mediante el test de Kolmogorov-Smirnov, indicando una distribución altamente sesgada y la necesidad de utilizar pruebas no paramétricas. El método elegido fue el modelo basado en los árboles 
de clasificación (Morgan y Sonquist, 1963). Se empleó el método de crecimiento del árbol CHAID (detector de interacción automática de Chi-cuadrado, aplicado en SPSS ${ }^{\mathrm{TM}}$ Answer Tree ${ }^{\odot}$ 13.0) ya que permite construir árboles no binarios, es decir, que incluyan más de dos ramas o divisiones de datos según categorías a explicar en cada nodo. Se aplicó un sistema de validación cruzada, siendo 50 el número mínimo de sujetos en los nodos terminales y 3 niveles máximos de árbol. Se consideraron 3 variables predictoras: 1) emoción (alegría, humor, amor, felicidad, ira, miedo, ansiedad, tristeza, vergüenza, rechazo, esperanza, sorpresa y compasión); 2) género (femenino, masculino); y 3) competición (presencia, ausencia). Se utilizó también el paquete estadístico Statistical Package for the Social Sciencies (SPSS) versión 19.0 para Windows.

\section{Resultados}

\section{Estudio 1: La emergencia de conflictos motores en los juegos de oposición}

El primer estudio examinó los 77 conflictos motores que emergieron en los 32 juegos de oposición que se propusieron durante la experiencia pedagógica. De estos juegos, debido a los objetivos de la experiencia pedagógica (reducción de la violencia y del absentismo escolar), un $62.5 \%$ (20) fueron propuestos con ausencia de competición, mientras que el $37.5 \%$ (12) restante se presentó con presencia de competición.

COMPORTAMIENTO DE LA VARIABLE COMPETICIÓN Y LA VARIABLE GÉNERO

Atendiendo a la variable competición, variable interna del juego motor, los datos muestran que surgieron conflictos motores en un $81.8 \%$ (63) cuando los juegos de oposición eran con ausencia de competición, mientras que surgieron en un $18.2 \%$ (14) cuando fueron con presencia de competición $(M=1.82 ; D T=.388)$.

Teniendo en cuenta la variable género, variable externa del juego motor, los conflictos motores que aparecieron en los juegos de oposición fueron generados en un $80.5 \%$ (62) por chicos, mientras que el $19.5 \%$ (15) restante fue originado por chicas $(M=1.81 ; D T=.399)$. Asimismo, la respuesta ante el estímulo de la conducta motriz conflictiva fue en un 58.4 $\%$ (45) originado por los chicos, mientras que las chicas lo hicieron en un 
¿Por qué te peleas? Conflictos motores y emociones negativas en la clase de Educación Física: el caso de los juegos de oposición

Unal Sáez de Ocáriz Granja, Pere Lavega Burgués, Francisco lagardera Otero, Antoni Costes Rodríguez y Jorge Serna Bardavío

$41.6 \%(32)(M=1.58 ; D T=.496)$. De esta manera, el género del conflicto motor en los juegos de oposición fue en un $51.9 \%$ (40) masculino, entre chicos; en un $14.3 \%$ (11) femenino, entre chicas; y en un $33.8 \%$ (26) mixto, entre chicos y chicas $(M=2.19$; $D T=.670)$.

Tras aplicar la prueba estadística de regresión logística para estudiar la relación existente entre el género del conflicto motor en los juegos de oposición y las respuestas conflictivas se puede afirmar $(\mathrm{p}<.05)$ las siguientes relaciones significativas (ver Tabla 3):

a) cuando los conflictos motores en los juegos de oposición fueron masculinos, entre chicos, emergieron más agresiones mixtas y menos agresiones verbales.

b) cuando los conflictos motores en los juegos de oposición fueron mixtos, entre chicos y chicas, se produjeron más agresiones verbales y menos agresiones mixtas.

Tabla 3. Tabla de contingencia entre el género del conflicto motor y la respuesta conflictiva

\begin{tabular}{llccc}
\hline \multirow{2}{*}{ GÉNERO DEL CONFLICTO MOtOR } & \multicolumn{3}{c}{ RESPUESTA CONFLICTIVA } \\
\cline { 3 - 5 } & & $\begin{array}{c}\text { Agresión } \\
\text { Verbal }\end{array}$ & $\begin{array}{c}\text { Agresión } \\
\text { Física }\end{array}$ & $\begin{array}{c}\text { Agresión } \\
\text { Mixta }\end{array}$ \\
\hline \multirow{2}{*}{ Masculino } & Recuento & 8 & 16 & 16 \\
& Residuos Corregidos & -3.4 & .4 & 2.0 \\
\multirow{2}{*}{ Femenino } & Recuento & 3 & 4 & 4 \\
& Residuos Corregidos & -.4 & -.1 & .5 \\
\multirow{2}{*}{ Mixto } & Recuento & 14 & 9 & 3 \\
& Residuos Corregidos & 2.9 & -.4 & -2.5 \\
\hline
\end{tabular}

\section{Estudio 2: la intensidad de las emociones negativas en los juegos de oposición}

El segundo estudio consideró 22246 datos sobre la intensidad emocional que experimentaron los participantes en los juegos de oposición. Se generaron unos árboles de clasificación con 54 nodos que fueron ordenados jerárquicamente en categorías homogéneas correspondientes a la relación entre las 13 variables independientes y la variable independiente (intensidad de la emoción) (ver Figura 1 y 2). 
La técnica de segmentación jerárquica identificó diferencias significativas $(p<.001)$ entre los 13 tipos de emociones. La ira/compasión registraron las intensidades más altas $(M=1.80)$, mientras que el resto de emociones se comportaron de una forma similar (ver Tabla 4).

Tabla 4. Comentarios relacionados con la intensidad de las emociones suscitadas en los juegos de oposición

\begin{tabular}{lllll}
\hline EMOCIÓN & $N$ & $\%$ & $M$ & $D T$ \\
\hline Alegría & 1716 & 7.7 & 7.11 & 2.48 \\
Humor & 1713 & 7.7 & 6.92 & 2.59 \\
Amor & 1712 & 7.7 & 3.33 & 3.13 \\
Felicidad & 1713 & 7.7 & 5.98 & 2.97 \\
Ira; Compasión & 3420 & 15.3 & 1.80 & 2.73 \\
Miedo & 1710 & 7.7 & .940 & 1.96 \\
Ansiedad & 1711 & 7.7 & 2.06 & 2.71 \\
Tristeza & 1711 & 7.7 & 1.12 & 2.15 \\
Vergüenza & 1709 & 7.7 & .703 & 1.65 \\
Rechazo & 1710 & 7.7 & .539 & 1.60 \\
Esperanza & 1711 & 7.7 & 4.10 & 3.39 \\
Sorpresa & 1710 & 7.7 & 3.78 & 3.35 \\
\hline
\end{tabular}

COMPORTAMIENTO DE LAS EMOCIONES NEGATIVAS

Del total de datos, el $53.8 \%$ (11971) hicieron referencia a la intensidad de las emociones negativas que emergieron en los juegos de oposición: ira, miedo, ansiedad, tristeza, vergüenza y rechazo (ver Figuras 1 y 2).

En relación a la ira se encontraron diferencias significativas entre los juegos de oposición con presencia y ausencia de competición $(p<.001)$. El género de los participantes fue la principal variable predictora en ambas situaciones $(p=.001$, con presencia de competición; $p<.001$, con ausencia de competición). En los juegos de oposición con presencia de competición las emociones más intensas aparecieron en los chicos $(M$ $=2.33)$, seguidas de las chicas $(M=1.82)$; mientras que en los juegos de oposición con ausencia de competición las emociones más intensas aparecieron en los chicos $(M=1.78)$, seguidas de las chicas $(M=1.28)$.

Teniendo en cuenta el miedo se encontraron diferencias significativas entre los juegos de oposición con presencia y ausencia de competición $(p<.001)$. El género de los participantes fue la principal variable predic- 
¿Por qué te peleas? Conflictos motores y emociones negativas en la clase de Educación Física: el caso de los juegos de oposición

Unal Sáez de Ocáriz Granja, Pere Lavega Burgués, Francisco lagardera Otero, Antoni Costes Rodríguez y Jorge Serna Bardavío

tora en los juegos de oposición con presencia de competición $(p=.001)$, donde las emociones más intensas aparecieron en los chicos $(M=.932)$, seguidas de las chicas $(M=.558)$. En los juegos de oposición con ausencia de competición no se detectó ninguna variable predictora.

En referencia a la ansiedad se encontraron diferencias significativas entre los juegos de oposición con presencia y ausencia de competición $(p=.032)$. El género de los participantes fue la principal variable predictora en los juegos de oposición con presencia de competición $(p=.002)$, donde las emociones más intensas aparecieron en los chicos $(M=2.50)$, seguidas de las de las chicas $(M=1.87)$. En los juegos de oposición con ausencia de competición no se detectaron variables predictoras.

Relacionado con la tristeza se encontraron diferencias significativas entre los juegos de oposición con presencia y ausencia de competición $(p<.001)$. El género de los participantes fue la principal variable predictora en ambas situaciones ( $p=.038$, con presencia de competición; $p$ $=.012$, con ausencia de competición). En los juegos de oposición con presencia de competición las emociones más intensas aparecieron en los chicos $(M=1.86)$, seguidas de las chicas $(M=1.47)$; mientras que en los juegos de oposición con ausencia de competición las emociones más intensas aparecieron en los chicos $(M=.783)$, seguidas de las chicas $(M=.522)$.

Teniendo en cuenta la vergüenza se encontraron diferencias significativas entre los juegos de oposición con presencia y ausencia de competición $(p<.001)$. No se detectaron variables predictoras en ninguna de las dos situaciones.

En relación al rechazo se encontraron diferencias significativas entre el género de los participantes $(p<.001)$. La competición fue la principal variable predictora en el caso de los chicos $(p=.004)$, donde las emociones más intensas aparecieron en los juegos de oposición con ausencia de competición ( $M=.056$ ), seguidas de las que emergieron en los juegos de oposición con presencia de competición $(M=.895)$. 
¿Por qué te peleas? Conflictos motores y emociones negativas en la clase de Educación

Física: el caso de los juegos de oposición Unai Sáez de Ocáriz Granja, Pere lavega Burgués, Francisco lagardera Otero, Antoni Costes Rodríguez y Jorge Serna Bardavío
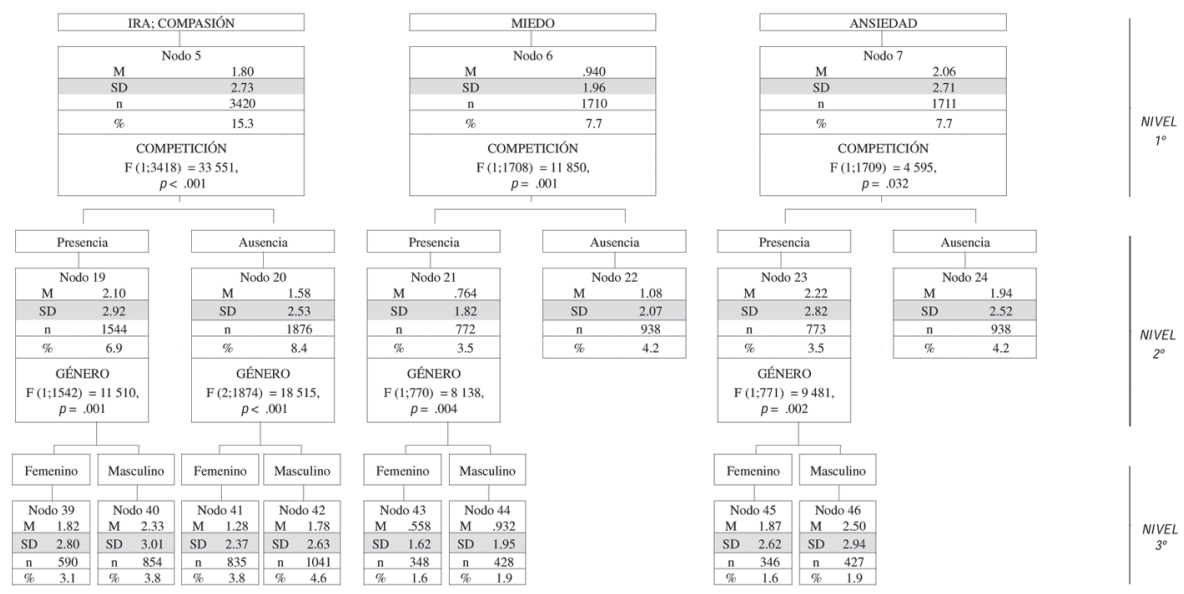

Figura 1. Variables predictoras en relación a la ira/compasión, miedo y ansiedad
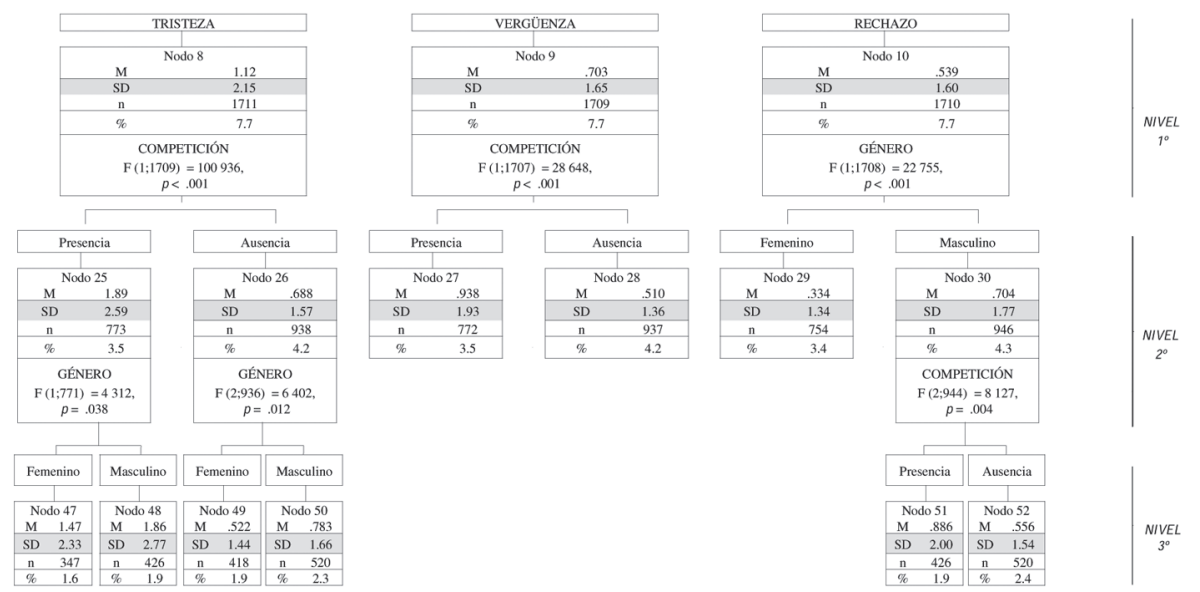

Figura 2. Variables predictoras en relación a la tristeza, vergüenza y rechazo

\section{Discusión}

El presente artículo examinó mediante dos investigaciones el efecto de la competición en los juegos de oposición (variable interna asociada al juego) y del género (variables externa asociada a los rasgos del alumno) en la aparición de conflictos motores y en la vivencia de emociones en el ámbito escolar. 
¿Por qué te peleas? Conflictos motores y emociones negativas en la clase de Educación Física: el caso de los juegos de oposición

Unal Sáez de Ocáriz Granja, Pere Lavega Burgués, Francisco lagardera Otero, Antoni Costes Rodríguez y Jorge Serna Bardavío

El primer estudio correspondió a una experiencia pedagógica dirigida a la reducción de la violencia y del absentismo escolar. Al emplear juegos de oposición para educar las relaciones interpersonales los alumnos confirman su elevado perfil conflictivo; se trata de personas socializadas en ambientes conflictivos en la que las demás personas se ven como rivales y no como compañeros de esa experiencia lúdica. Con estos alumnos se observan más conflictos motores en juegos motores sin competición; a pesar de ser una situación contradictoria, se debe considerar que la oposición y el modelo "ganar-perder" forman parte activa de su realidad social, donde los roles de ganador y perdedor adquieren una gran importancia.

Cuando no queda definido el éxito y el fracaso en los juegos de oposición sin competición se pueden originar situaciones de miedo, inseguridad y frustración al tener que dar respuesta a situaciones a las que no se está habituado (Rubin, Chen, y Hymel,1993).

Los juegos de oposición originan en estos alumnos altos niveles de impulsividad lo que dificultan el control de la agresividad (Olweus, 1993), que, junto a sus escasas habilidades en la gestión de emociones, favorece la emergencia de conductas antisociales conflictivas (Carney y Merrel, 2001; Fernández-Berrocal y Ruiz-Aranda, 2008; Mestre et al., 2006; Petrides et al., 2004).

Por otro lado, desde la perspectiva de género se observa que los chicos protagonizan mayor cantidad de conflictos, acompañados de respuestas conflictivas de mayor intensidad, agresiones físicas y verbales simultáneas. Estos resultados van en la línea de diferentes estudios realizados en Europa (Cerezo, 2009; Defensor del Pueblo, 2007; Díaz-Aguado, 2005; García-Correa, 2001; Ortega, 2002).

En el segundo estudio los datos revelan que las variables competición y género de los participantes pueden predecir la emergencia de emociones negativas. La presencia o ausencia de competición es la primera variable predictiva de las emociones ira, miedo, ansiedad, tristeza y vergüenza. Además en algunos casos, saber si intervienen chicos o chicas es la segunda variable predictiva de estas emociones negativas. Esta diferencia se podría deber a la diferencias que existe en la socialización lúdica y social de los estereotipos de género. Los hallazgos de esta segunda investigación confirman lo observado en otros estudios al indicar que la derrota puede generar emociones negativas como la ira, la tristeza e incluso el rechazo (Hanin, 2000; Lavega et al., 2013; Lazarus, 
2000). Asimismo, corroboran los datos ofrecidos por Alonso et al. (2013) al afirmar que las emociones negativas son más intensas cuando existe competición. En este sentido, adquieren coherencia las apreciaciones realizadas por Lavega et al. (2011) quienes sugieren introducir juegos motores sin competición para evitar clasificar al alumnado y reducir la presencia de emociones negativas.

\section{Conclusiones}

Tal y como muestran los resultados ofrecidos en las investigaciones sobre la emergencia de conflictos motores y la intensidad de las emociones negativas, se puede indicar que la competición (variable interna) y el género de los protagonistas (variable externa) conviene tenerlas muy en cuenta si se pretende abordar el tratamiento pedagógico de los conflictos motores y regular la intensidad de las emociones negativas en los juegos de oposición.

Asimismo, tal como sugieren otros autores (e.g., Bell y Song, 2005; Bisquerra, 2000; Desivilya y Yagil, 2005; Deutsch, Coleman, y Marcus, 2006; Jameson, Bodtker, Porch, y Jordan, 2009; Lazarus, 2000), se puede apreciar la vinculación existente entre los conflictos motores y la intensidad de las emociones negativas, y cómo dichos conflictos pueden emerger cuando los protagonistas no son capaces de regular sus emociones negativas.

Los juegos de oposición constituyen una familia singular de situaciones de aprendizaje de las relaciones interpersonales. Cuando los alumnos tienen suficientes competencias de relación social estas experiencias activan de manera considerable la intensidad emocional. Sin embargo, ante un grupo con problemas de convivencia son juegos que pueden acentuar los conflictos ya que el rival se suele ver como un agente hostil para la relación interpersonal.

Además al profesor de EF le interesa saber que en la práctica de estos juegos los chicos generan mayor cantidad de conflictos que las chicas y también mayor intensidad de emociones negativas, sobre todo si se juega a competir.

Todas estas aportaciones ofrecen al educador evidencias sobre aqueIlos aspectos que conviene considerar al introducir los juegos de oposición en sus programas de intervención. 
¿Por qué te peleas? Conflictos motores y emociones negativas en la clase de Educación Física: el caso de los juegos de oposición

Unai Sáez de Ocáriz Granja, Pere lavega Burgués, Francisco lagardera Otero, Antoni

Costes Rodríguez y Jorge Serna Bardavío

Por último, indicar la limitación que suponen los resultados mostrados al provenir de dos estudios diferentes. En un futuro se plantea estudiar con los mismos protagonistas el efecto de la competición de los juegos de oposición y la perspectiva de género en los conflictos motores $y$ en vivencia emocional.

\section{Referencias bibliográficas}

Alonso, J.I., Etxebeste, J., \& Lavega, P. (2010). Análisis de emociones suscitadas en juegos motores con y sin competición. En VI Congreso Internacional de la Asociación Española de Ciencias del Deporte, Elche: Universidad Miguel Hernández.

Alonso, J.I., Gea, G., \& Yuste, J.L. (2013). Formación emocional y juego en futuros docentes de Educación Física. Revista Electrónica Interuniversitaria de Formación del Profesorado, 16(1), 97-108.

Alonso, J.I., Lavega, P., \& Reche, F. (2012). Análisis de la vivencia emocional desde la perspectiva de género en juegos deportivos de cooperación con oposición en alumnos de Bachillerato. En Martínez, Cayero \& Calleja (Coord.). Investigación e innovación en el deporte (pp. 161-169). Barcelona: Paidotribo.

Baron, R. A. (1990). Enviromentally induced positive affect: its impact on self-efficacy, task performance, negotiation, and conflict. Journal of Applied Social Psychology, 20(5), 368-384. doi: 10.1111/j.1559-1816.1990.tb00417.x

Bell, C., \& Song, F. (2005). Emotions in the conflict process: An application of the cognitive appraisal model of emotions to conflict management. The International Journal of Conflict Management, 16(1), 30-54.

Bisquerra, R. (2000). Educación emocional y bienestar. Barcelona: Praxis.

Bodtker, A. M., \& Jameson, J. K. (2001). Emotion in conflict formation and its transformation: Application to organizational conflict management. The International Journal of Conflict Management, 12(3), 259-275.

Burguet, M. (2003). Ante el conflicto. Una propuesta por la educación. En E. Vinyamata (Coord.), Aprender del conflicto. Conflictología y educación. Barcelona: Graó.

Carney, A. G., \& Merrel, K. W. (2001). Bullying in schools: perspectives on understanding and preventing an international problem. School Psychology International, 22(3), 364-382. doi:10.1177/0143034301223011

Cerezo, F. (2009). Bullying: Análisis de la situación en las aulas españolas. International Journal of Psychology and Phychological Therapy, 9(3), 383-394.

Cervelló, E., Moreno, J. A., Alonso, N., \& Iglesias, D. (2006). Goal orientations, motivational climate and dispositional flow of high school students engaging in extracurricular involvement in physical activity. Perceptual and Motor Skills, 102, 87-92.

Costes, A. (1997). El profesor de educación física como investigador. Revista Electrónica Interuniversitaria de Formación del Profesorado, 1(0), 1-4.

Defensor del Pueblo. (2007). Violencia escolar: el maltrato entre iguales en la educación secundaria obligatoria 1999-2006. Madrid: Defensor del Pueblo. 
¿Por qué te peleas? Conflictos motores y emociones negativas en la clase de Educación

Física: el caso de los juegos de oposición

Unal Sáez de Ocáriz Granja, Pere lavega Burgués, Francisco lagardera Otero, Antoni

Costes Rodríguez y Jorge Serna Bardavío

Del Rincón, D., Arnal, J., Latorre, A., \& Sans, A. (1995). Técnicas de investigación en ciencias sociales. Madrid: Editorial Dykinson.

Desivilya, H. S., \& Yagil, D. (2005). The role of emotions in conflict management: The case of work teams. The International Journal of Conflict Management, 16(1), 55-69. doi: 10.1108/eb022923

Deutsch, M., Coleman, P., \& Marcus, E. (2006). The handbook of conflict resolution. Theory and practice (2nd ed.). San Francisco: Jossey-Bass.

Díaz-Aguado, M. J. (2005). La violencia entre iguales en la adolescencia y su prevención desde las aulas. Psicothema, 17(4), 549-558.

Digelidis, N., Papaioannou, A., Laparidis, K., \& Christodoulidis, T. (2003). A one-year intervention in 7 th grade physical education classes aiming to change motivational climate and attitudes toward exercise. Psychology of Sport and Exercise, 4, 195-210.

Elías, N. (1987). El proceso de civilización. Investigaciones sociogenéticas y psicogenéticas. Madrid: Fondo de Cultura Económica.

Fernández-Berrocal, P., \& Ruiz-Aranda, D. (2008). La inteligencia emocional en la educación. Revista Electrónica de Investigación Psicoeducativa, 6(2), 421-436.

García-Correa, A. (2001). La violencia en centros educativos de Norteamérica y diferentes países de Europa. Medidas políticas tomadas para la convivencia escolar. Revista Interuniversitaria de Formación de Profesorado, 41, 167-177.

Hanin, Y.L. (2000). Emotions in sport. Champaign. Illinois: Human Kinetics.

Jameson, J. K., Bodtker, A. M., Porch, D. M., \& Jordan, W. J. (2009). Exploring the role of emotion in conflict transformation. Conflict Resolution Quaterly, 27(2), 167-192. doi:10.1002/crq.254

Jiménez, R., Cervelló, E., Santos-Rosa, F. J., García-Calvo, T., \& Del Villar, F. (2006). Un estudio de las relaciones entre las metas de logro, la percepción del clima motivacional, la valoración de la educación física, la práctica deportiva extraescolar y el consumo de drogas en estudiantes de educación física. Revista Mexicana de Psicología, 23, 253-265.

Lagardera, F., \& Lavega, P. (2005). La educación física como pedagogía de las conductas motrices. Tándem. Didáctica de la Educación Física, 18, 79-102.

Lagardera, F., \& Lavega, P. (2011). Educación Física, conductas motrices y emociones. Revue Ethologie \& Praxeologie, 16, 23-44.

Lavega, P. (2004). Aplicaciones de la noción de conducta motriz en la enseñanza. En F. Lagardera \& P. Lavega (Eds.), La ciencia de la acción motriz (pp. 157-180). Lleida: Ediciones de la Universitat de Lleida.

Lavega, P., Filella, G., Agullo, M.J., Soldevilla, A., \& March, J. (2011). Conocer las emociones a través de juegos: Ayuda para los futuros docentes en la toma de decisiones. Electronic Journal of Research in Educational Phychology, 9(2), 617-640.

Lavega, P., Filella, G., Lagardera, F., Mateu, M., y Ochoa, J. (2013). Juegos motores y emociones. Cultura y Educación, 25(3), 347-360.

Lavega, P., March, J., \& Filella, G. (2013). Juegos deportivos y emociones. Propiedades psicométricas de la escala GES para ser aplicada en la Educación Física y el Deporte. Revista de Investigación Educativa, 31(1), 151-166. 
¿Por qué te peleas? Conflictos motores y emociones negativas en la clase de Educación Física: el caso de los juegos de oposición

Unai Sáez de Ocáriz Granja, Pere lavega Burgués, Francisco lagardera Otero, Antoni

Costes Rodríguez y Jorge Serna Bardavío

Lazarus, R.S. (2000). How emotions influence performance in competitive sports. The Sport Psychologist, 14, 229-252.

Lederach, J. P. (1995). Preparing for peace. Conflict transformation across cultures. New York: Syracuse University Press.

Lee, C.C., Mower, E., Busso, C., Lee, S., \& Narayanan, S. (2011). Emotions recognition using hierarchical binary decision tree approach. Speech Communication, 53, 1162-1171.

Mesía, R. (2007). Contexto ético de la investigación social. Investigación Educativa, 11(19), 137-151.

Mestre, J. D., Guil, R., Lopes, P., Salovey, P., \& Olarte, G. (2006). Emotional intelligence and social and academia adaptation to school. Psicothema, 18, 112-117.

Morgan, J. N., \& Sonquist, J.A. (1963). Problems in the analysis of survey data, and a proposal. Journal of the American Statistical Association, 58, 415-434.

Newton, M., Duda, J. L., \& Yin, Z. (2000). Examination of the psychometric properties of the perceived motivational climate in sport questionnaire-2 in a sample of female athletes. Journal of Sports Sciences, 18, 275-290.

Olweus, D. (1993). Bullying at school: what we know and what we can do. Oxford: Blackwell.

Ortega, R. (2002). Lo mejor y lo peor de las redes de iguales: juego, conflicto y violencia. Revista Interuniversitaria de Formación de Profesorado, 44, 93-113.

París, S. (2005). La transformación de los conflictos desde la filosofía para la paz. Universitat Jaume I (Tesis Doctoral inédita), Castelló de la Plana. Recuperada de http://www. tdx.cat/bitstream/handle/10803/10456/paris.pdf?sequence $=1$

Parlebas, P. (2001). Juegos, deporte y sociedad. Léxico de praxiología motriz. Barcelona: Paidotribo.

Petrides, K. V., Frederickson, N., \& Furnham, A. (2004). The role of trait emotional inteIligence in academic performance and deviant behaviour at school. Personality and Individual Differences, 36(2), 277-293. doi:10.1016/S0191-8869(03)00084-9

Ramírez-Fernández, S. (2006). El maltrato entre escolares y conductas-problemas para la convivencia: un estudio desde el grupo-clase. Granada: Universidad de Granada.

Rubin, K. H., Chen, X., \& Hymel, S. (1993). Socioemotional characteristic of withdrawn and aggressive children. Merril-Palmer Quaterly, 39(4), 518-534.

Sáez de Ocáriz, U. (2011). Conflictos y educación física a la luz de la praxiología motriz. Estudio de caso de un centro educativo de primaria. Universitat de Lleida - INEFC Lleida (Tesis Doctoral inédita), Lleida. Recuperada de http://www.tdx.cat/handle/10803/53637

\section{Agradecimientos}

Estos estudios han recibido financiación del Ministerio de Economía y Competitividad, Secretaría de Estado de Investigación, Desarrollo e Innovación de España a través del proyecto I+D+i Juegos Deportivos Psicomotores y de Cooperación DEP2010-21626-C03-01. 